\title{
Runoff generation dynamics within a humid river basin
}

\author{
S. Manfreda \\ Dipartimento di Ingegneria e Fisica dell'Ambiente (DIFA), Università degli Studi della Basilicata, via dell' Ateneo Lucano, \\ 10, Potenza, 85100, Italy
}

Received: 25 April 2008 - Revised: 1 October 2008 - Accepted: 27 October 2008 - Published: 8 December 2008

\begin{abstract}
The present paper introduces an analytical approach for the description of the soil water balance and runoff production within a schematic river basin. The model is based on a stochastic differential equation where the rainfall is interpreted as an additive noise in the soil water balance and is assumed uniform over the basin, the basin heterogeneity is characterized by a parabolic distribution of the soil water storage capacity and the runoff production occurs for saturation excess. The model allowed to derive the probability density function of the produced surface runoff highlighting the role played by climate and physical characteristics of a basin on runoff dynamics. Finally, the model have been tested over a humid basin of Southern Italy proposing also a strategy for the parameters estimation.
\end{abstract}

\section{Introduction}

Runoff production mechanisms are influenced by several factor such as: the condition of the soil surface and its vegetative cover, the soil texture, and the antecedent soil moisture content. The role of each of those factors may change according to the specific climatic conditions. In particular, Hortonian runoff production mechanism (Horton, 1933) for describing storm runoff is more suitable for arid region, where infiltration capacity is generally lower. In most humid regions, permeability of the soil is high because the vegetation cover protects the soil from rain packing and creates an open soil structure. Under such conditions, rainfall intensities generally do not exceed infiltration capacities and consequently Hortonian overland flow does not occur. In humid environment, the variable source area concept, first introduced by Hewlett and Hibbert (1967), is widely accepted to explain

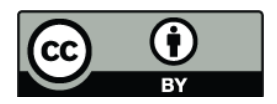

Correspondence to: S. Manfreda (manfreda@unibas.it) storm runoff production through return flow and direct precipitation. The variable source area represent the saturated portion of the basin that vary dynamically according to the soil moisture state of the basin.

Recently, Manfreda and Fiorentino (2008) developed a new model aimed at the definition of the probability distribution of the relative saturation of a basin characterized by a parabolic distribution of the soil water storage capacity. The model stems from previous works carried out, using a stochastic differential equation, to describe the soil moisture dynamics at the point scale (e.g., Rodríguez-Iturbe et al., 1999; Laio et al., 2001; Porporato et al., 2004). The proposed scheme includes a number of approximations, but it leads to an interesting framework for the derivation of the main statistics of basin scale variables. Among others, our interest focused on the behavior of saturated areas over a basin that may be responsible of the dynamics of runoff generation.

The theory is based on the conceptual model Xinanjiang that describes watershed heterogeneity using a parabolic curve for the distribution of the water storage capacity (Zhao et al., 1980). The Xinanjiang model is a well-known lumped watershed model widely used in China. Furthermore, the proposed relationship between the extent of saturated areas and the volume of water stored in the catchment has driven the evolution of a number of more recent models such as the Probability Distributed Model (Moore and Clarke, 1981; Moore, 1985, 1999), the VIC model (Wood et al., 1992, 1997; Liang et al., 1994) and the ARNO model (Todini, 1996).

The analytical approach used in the present research offers the advantage of providing general class of solutions and significant results of wide applicability, although obviously limited by the unavoidable simplifications adopted to solve the mathematical problem analytically. The paper provides a description of the model characteristics introduced by Manfreda and Fiorentino (2008) in Sect. 2. The theoretical derivation of the probability distribution of the runoff

Published by Copernicus Publications on behalf of the European Geosciences Union. 
production is given in the Sect. 3. Results of the model are discussed in Sect. 4 that precedes the conclusions.

\section{Model description}

\subsection{Rainfall model}

Rainfall occurrences are modelled by a sequence of instantaneous pulses that occur in a Poisson process of rate $\lambda$ in time. Each pulse is characterized by a random total depth $Y$ exponentially distributed with mean $m_{Y}=\alpha$ that may be considered as the mean daily rainfall since the model is interpreted at the daily time-scale (see Rodríguez-Iturbe et al., 1999).

The normalized version of the density function of rainfall depths can be described as

$f_{H}(Y)=\gamma e^{-\gamma Y}$

where $\gamma=w_{\max } / \alpha$ and $w_{\max }$ is the maximum value of the water storage capacity in the basin.

The spatial heterogeneity of rainfall is neglected assuming uniform distribution of rainfall occurring at random in time over the entire basin. Such an assumption may be more or less reliable depending on climatic characteristics of the area and the basin size. In general, this hypothesis becomes more realistic for river basins of medium/small sizes and in humid regions.

2.2 The variability of the soil water storage capacity over the basin

The soil thickness is assumed to vary over the basin according to a given distribution. In fact, the heterogeneity in the soil water storage capacity was observed to be a controlling factor in the temporal dynamics of soil moisture (Manfreda and Rodríguez-Iturbe, 2006). For sake of simplicity, the remaining sources of heterogeneity like pattern of vegetation and soil texture variability have been neglected assuming that the soil texture as well as the vegetation are uniform over the watershed.

The watershed heterogeneity is described using a parabolic curve for the water storage capacity of the soil (Zhao et al., 1980)

$\frac{f}{F}=1-\left(1-\frac{W}{w_{\max }}\right)^{b}$

where $f / F$ represents the fraction of the basin with water storage capacity $\leq W, w_{\max }$ represents the maximum value of the water storage capacity in the basin and $b$ is a shape parameter that according to Zhao (1992) assumes values between 0.1-0.4 increasing with the characteristic dimension of the basin. The parameter $b$ controls the spatial variability of $W$ that increases with larger values of $b$ and becomes uniform when $b=0$.
The total water storage capacity of the basin is obtained integrating $(1-f / F)$ between $W=0$ and $w_{\max }$, obtaining

$W M=\frac{w_{\max }}{1+b}$.

In order to obtain a water balance equation with only one state variable, it is necessary to make the hypothesis that the soil water distribution is known over the basin. In particular, it is possible to assume that the soil water content is redistributed within the basin cumulating in the areas with lower soil water storage capacity following the same schematization abopted within the Xinanjiang model. The conceptual schematization of the basin is sketched in Fig. 1, where both the soil water content distribution and the soil water capacity are described. From this graph, it is also clear that the relative saturated areas, $a$, are described by the same relationship given in Eq. (2) where $a$ correspond to the ratio $f / F$.

The watershed-average soil moisture storage at time $t$, is the integral of $1-f / F$ between zero and the actual value of the water level in the basin scheme, $w m_{t}$,

$W_{t}=W M\left(1-\left(1-\frac{w m_{t}}{w_{\max }}\right)^{1+b}\right)$.

Under the described schematization, the relative saturation of the basin, $s$, expressed as the ratio between watershedaverage soil moisture storage and the total available volume can be defined as

$s=\frac{W_{t}}{W M}=\left(1-\left(1-\frac{w m_{t}}{w_{\max }}\right)^{1+b}\right)$.

\subsection{The soil water losses}

The function describing the soil water losses represents the deterministic part of the stochastic equation describing the soil water balance. It depends on the local value of the soil water content and the maximum rate of soil water losses. The main contributions to soil losses are given by: the actual evapotranspiration and the soil leakage. A possible approximation for the sum of this two terms is given by a linear function where the soil loss is assumed to be proportional to the soil water content (see e.g. Entekhabi and Rodríguez-Iturbe, 1994; Pan et al., 2003; Isham et al., 2005)

$L(\zeta)=V \zeta(t, x)$,

where $L(\zeta)$ is the soil water loss relative to the soil saturation $\zeta(t, x)$ at time $t$ in the point $x$ in space, and $V$ is the water loss coefficient.

The described linear equation was used in several analytical and experimental studies. Among others, Pan et al. (2003) defined an expression to estimate the values of $V$ as a function of the soil permeability, $K_{S}$, and Leaf Area Index (LAI). They obtained two different expressions in the two considered study cases

$$
\begin{aligned}
& V=\max \left(1,12.27+1.06 K_{s}-0.88 \mathrm{LAI}\right) \text { Monsoon' }^{\prime} 90 \\
& V=\max \left(1,6.08+0.40 K_{s}-0.51 \mathrm{LAI}\right) \quad \text { Washita'}^{\prime} 92
\end{aligned}
$$



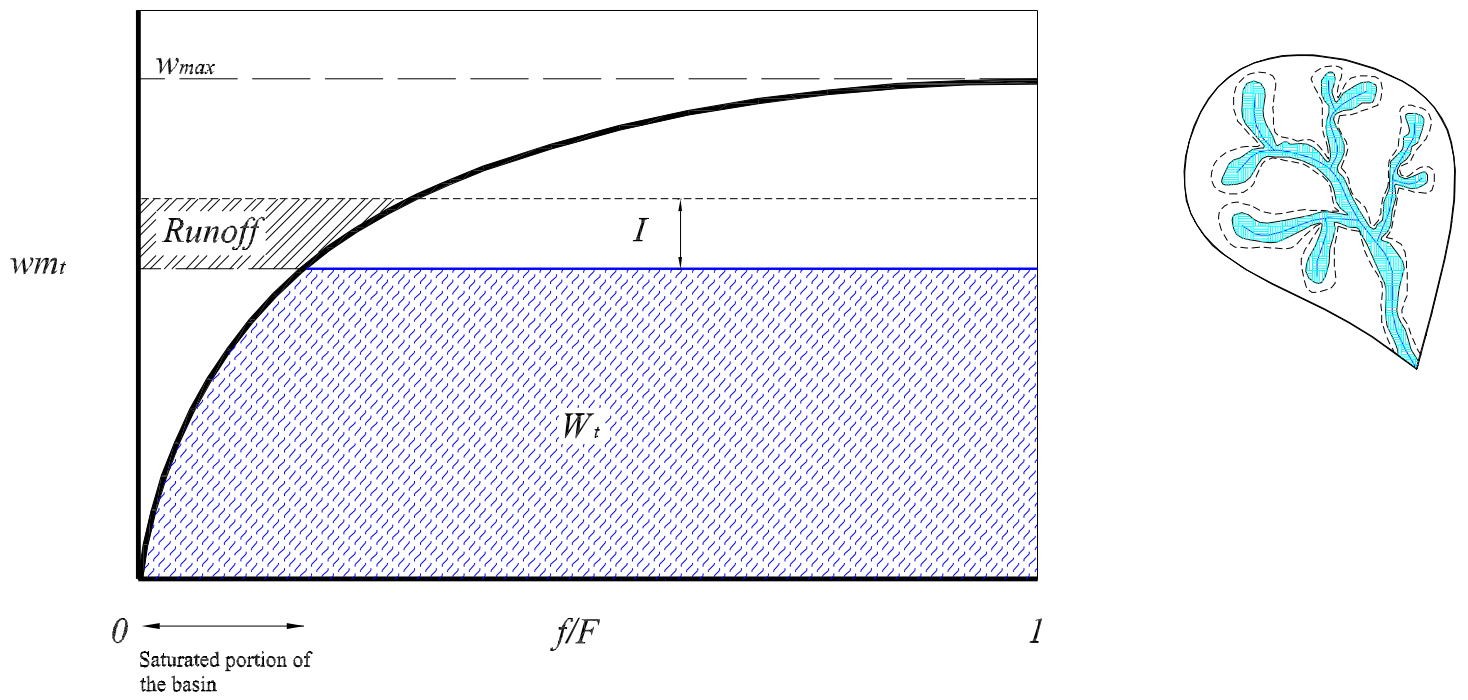

Fig. 1. Schematization of the basin structure and soil water content distribution. The black line represents the distribution of the soil water storage capacity, $W$, that ranges from 0 to $w_{\max }$; the blue line depicts the water distribution over the schematic basin whose level is $w m_{t}$; the dashed line depicts the increase in $w m_{t}$ after a rainfall event producing an infiltration $I$ over the unsaturated portion of the basin, while the saturated and the becoming saturated portion of the basin will produce a runoff represented by the dashed area of the graph.

where $V$ is expressed in $\mathrm{mm} /$ day and $K_{S}$ in $\mathrm{cm} / \mathrm{h}$. Among the two, the one derived from the Washita ' 92 data set was successfully tested by Manfreda and Fiorentino (2008) in a Mediterranean basin.

Since the adopted soil loss function is a linear one, it can be generalized at the basin scale using the product between the relative basin saturation, $s$, and the water loss coefficient. The soil water loss function at the basin scale becomes

$L_{b}\left(w m_{t}\right)=V s=V\left(1-\left(1-\frac{w m_{t}}{w_{\max }}\right)^{1+b}\right)$.

For analytical purposes, the soil water losses can be expressed as a function of the ratio $R=\frac{w m_{t}}{w_{\text {max }}}$ using an approximated expression in exponential form. In this case, the soil water losses are expressed as

$L_{b}\left(R=\frac{w m_{t}}{w_{\max }}\right) \cong V\left(\frac{e^{-k R}-1}{e^{-k}-1}\right)$

where $k$ is a coefficient that has been used to fit the above with Eq. (8). This yields $k \cong b /\left(\frac{b}{7}-\frac{1}{3}\right)$.

\subsection{The water balance equation}

The water balance equation can be written at the basin scale working with the water level in the parabolic reservoir that leads to the following stochastic differential equation in $w m_{t}$

$\frac{d w m_{t}}{d t}=I-V s$ where $I$ represents an additive term of infiltration and water losses are assumed to be proportional to the relative saturation of the basin $s$. The advantage to solve the water balance equation in $w m_{t}$ is that the infiltration rate can be summed as an additive term in the stochastic differential equation. After a rainfall event of depth $Y$, the water level $w m_{t}$ in the basin schematization increases of $Y$ as long as the rainfall depth plus the actual $w m_{t}$ does not exceed the maximum water storage capacity of the basin $w_{\max }$. Consequently, the infiltration $I$ is equal to the rainfall depth if $Y \leq\left(w_{\max }-w m_{t}\right)$ other wise becomes equal to $\left(w_{\max }-w m_{t}\right)$. The schematization, in fact, accounts for the upper bound imposed by the soil saturation.

In the present scheme, the runoff generation occurs when rainfall falls over a saturated portion of the basin. This behavior is comparable with a Dunne mechanism where the direct precipitation on saturated areas (saturated overland flow) is a dominant runoff generation mechanism (e.g., Hewlett and Hibbert, 1967; Dunne and Black, 1970).

The water balance equation can be solved using the standardized variable

$R=\frac{w m_{t}}{w_{\max }}$

where $R \in[0,1]$.

The water balance equation becomes

$\frac{d R}{d t}=\frac{Y}{w_{\max }}-\rho(R)$ 
where $\rho(R)$ is the standardized soil water loss rate

$\rho(R)=\beta\left(\frac{e^{-k R}-1}{e^{-k}-1}\right)$,

with $\beta=V /\left(w_{\max }\right)$, that represents the normalized soil water loss coefficient.

Following Rodríguez-Iturbe et al. (1999), the probability density function (PDF) of $R$ can be obtained and solved analytically for steady-state conditions. The PDF of $R$, obtained using the simplified loss function $\rho(R)$ in the water balance equation above, becomes

$$
\begin{aligned}
& p(R)=\frac{C}{\rho(R)} e^{-\gamma R+\lambda \int \frac{1}{\rho(R)} d u}= \\
& \frac{C e^{k(R-1)-R \gamma}\left(e^{k}-1\right)\left(e^{k R}-1\right)^{\frac{\lambda\left(1-e^{-k}\right)}{k \beta}-1}}{\beta},
\end{aligned}
$$

where $C$ is a constant of integration that may be computed simply imposing the normalizing condition, $\int_{0}^{1} p(R) d R=1$. Thus, $C$ assumes the following value

$$
\begin{aligned}
& C=1 / \int_{0}^{1} \frac{e^{k(-1+R)-R \gamma}\left(e^{k}-1\right)\left(e^{k R}-1\right)^{-1+\frac{\lambda-e^{-k} \lambda}{k \beta}}}{\beta} d R \\
& C=\frac{\beta}{\frac{\Gamma\left[1-\frac{\gamma}{k}\right] \Gamma\left[\frac{\lambda-e^{-k \lambda}}{k \beta}\right]}{k e^{k} \Gamma\left[\frac{k \beta-\beta \gamma+\lambda-e^{-k} \lambda}{k \beta}\right]}+\frac{F_{1}\left[1-\frac{\gamma}{k}, 1+\frac{\left(e^{-k}-1\right) \lambda}{k \beta}, 2-\frac{\gamma}{k}, e^{k}\right]}{e^{\gamma}(\gamma-k)}} \cdot \\
& (-1) \frac{1}{\frac{\lambda-e^{-k}}{k \beta}}\left(e^{k}-1\right)
\end{aligned}
$$

where $\Gamma[$.$] is the complete Gamma Function and F_{1}[.$, ., ., .] is the Hypergeometric Function (Abramowitz and Stegun, 1964).

The analytical expression of the probability density function of the standardized variable $R$ (Eq. 14) can be used to derive the probability distributions of the relative saturation and of the saturated areas of the basin. This is a straightforward operation when the functional relationships between these variables are determined. Manfreda and Fiorentino (2008) provide the analytical probability distribution for these two variables also describing the role played by the model parameters on the dynamics of saturated areas as well as relative saturation of the basin.

\section{Cumulative probability distribution of runoff}

In the present section, the cumulative probability distribution of runoff is derived within the context of the analytical framework traced in the previous paragraphs.

The runoff production mechanisms at the catchment scale are mostly controlled by the temporal variability and also by the spatial variability of rainfall processes (this last is neglected in the present study), and by the spatial distribution of soil moisture. In the proposed schematization, the runoff magnitude depends on both the state of the system (i.e. $R$ ) and the rainfall depth, $Y$. The runoff production can be described through the following equations

$$
\begin{array}{ll}
q= & \\
Y-\frac{w_{\max }\left((1-R)^{1+b}-\left(1-R-\frac{Y}{w_{\max }}\right)^{1+b}\right)}{1+b} ; & Y \leq(1-R) w_{\max } \\
Y-\frac{w_{\max }(1-R)^{1+b}}{1+b} ; & Y \geq(1-R) w_{\max }
\end{array}
$$

where the runoff mechanism switches from a partial contributing area to a total contributing area when $Y$ exceeds the threshold $(1-R) w_{\max }$ that is the rainfall amount required to saturate the soils with higher water storage capacity.

The above expressions can be inverted as a function of $Y$ using in the first case a Taylor expansion of the second order around zero, while the second expression can be inverted without any approximation. It follows that

$$
\begin{array}{ll}
Y= & \\
\frac{w_{\max }\left((1-R)^{b}-1+\sqrt{\frac{2 b q(1-R)^{b-1}}{w_{\max }}+\left((1-R)^{b}-1\right)^{2}}\right)}{b(1-R)^{b-1}} ; & Y \leq(1-R) w_{\max } \\
q+\frac{w_{\max }}{1+b}(1-R)^{1+b} ; & Y \geq(1-R) w_{\max }
\end{array}
$$

In order to derive the probability distribution of runoff, one should integrate the join probability distribution of rainfall, $Y$, and $R$ over the proper region of the 2-D space in $R$ and $Y$ where the runoff is lower than an assigned $q$ (e.g., Benjamin and Cornell, 1970). The CDF of the runoff assumes the following expression

$$
\begin{aligned}
& P_{Q}(q)= \\
& \int_{0}^{R_{1}} \int_{0}^{\frac{w_{\max }\left((1-R)^{b}-1+\sqrt{\frac{2 b q(1-R)^{b-1}}{w_{\max }}+\left((1-R)^{b}-1\right)^{2}}\right)}{b(1-R)^{b-1}}} p(R) g(Y) d Y d R \\
& +\int_{R_{1}}^{1} \int_{0}^{q+\frac{w_{\max (1-R)^{1+b}}}{1+b}} p(R) g(Y) d Y d R
\end{aligned}
$$

where

$$
R_{1} \simeq \frac{1}{6}\left(1-b+\frac{\sqrt{b(5+b)^{2} w_{\max }-12(1+b)(2+b)}}{\sqrt{b w_{\max }}}\right)
$$

is the value of $R$ at which there is a transition in the runoff production from a partial contributing area to a total contributing area. The integration scheme adopted to derive the cumulative probability distribution given above is described in Fig. 2 where the region of values of $R$ and $Y$ that involve values of runoff lower than $q$ is dashed with different symbols in order to differentiate among the two possible runoff mechanisms (partial contributing area and total contributing area). 


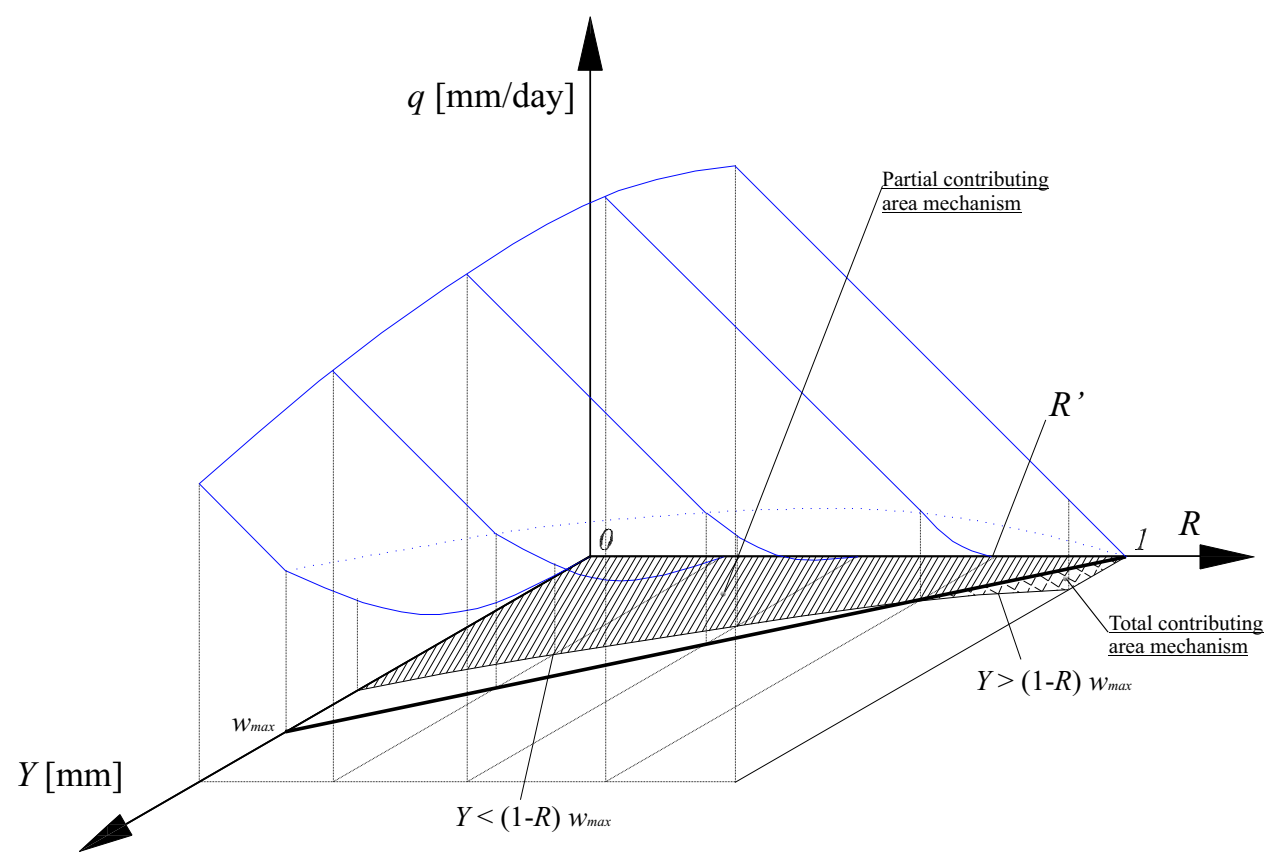

Fig. 2. Description of runoff as a function of the rainfall depth, $Y$, and the state of the basin described by the ratio $R=\frac{w m_{t}}{w_{\max }}$.
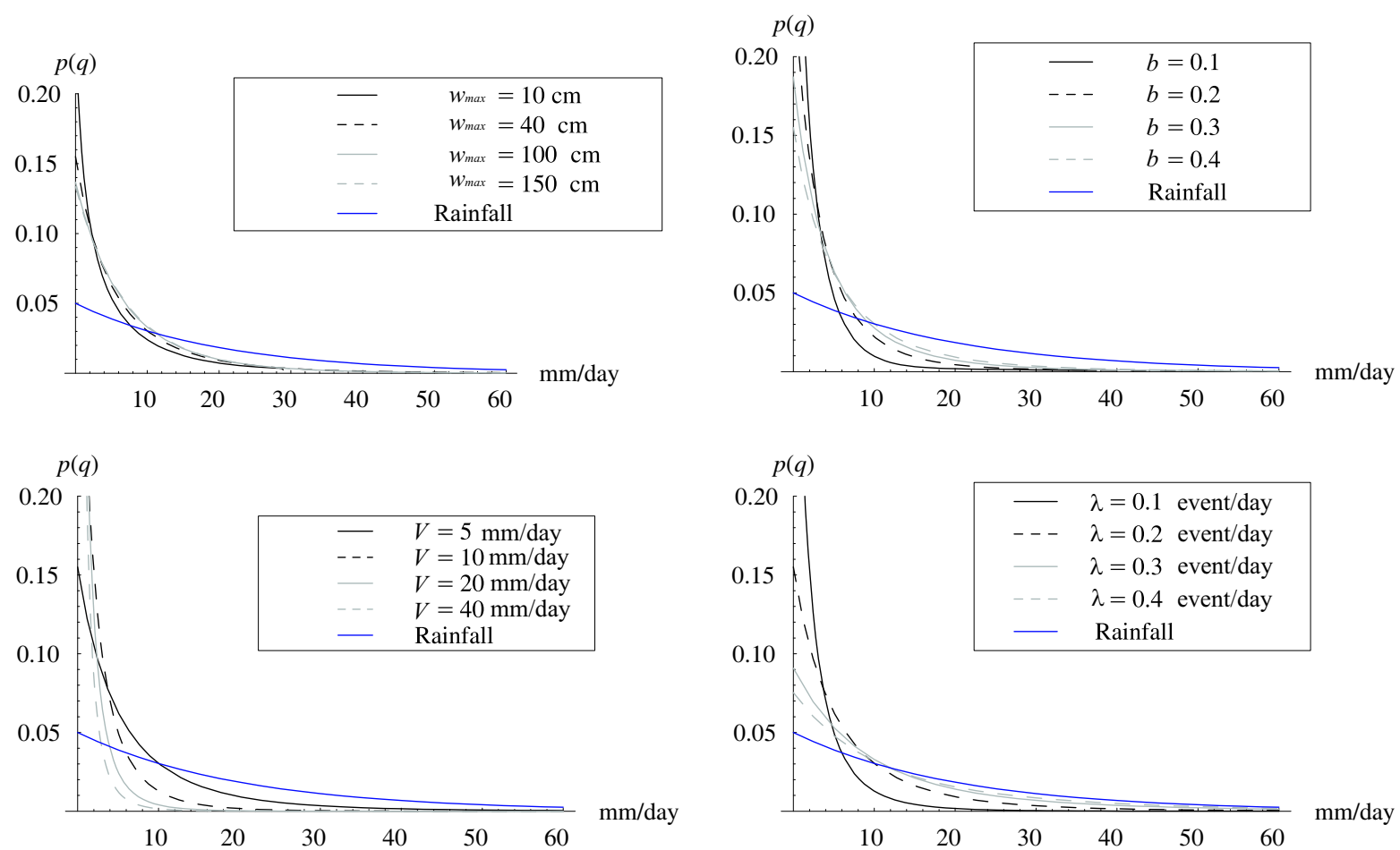

Fig. 3. Probability density functions of runoff and rainfall obtained varying the parameters: $w_{\max }$ (top-left), $b$ (top-right), $V$ (bottom-left) and $\lambda$ (bottom-right). The reference parameters are $V=5 \mathrm{~mm} / \mathrm{day}, w_{\max }=40 \mathrm{~cm}, b=0.4, \alpha=20 \mathrm{~mm}$ and $\lambda=0.2$ event $/ \mathrm{day}$. 

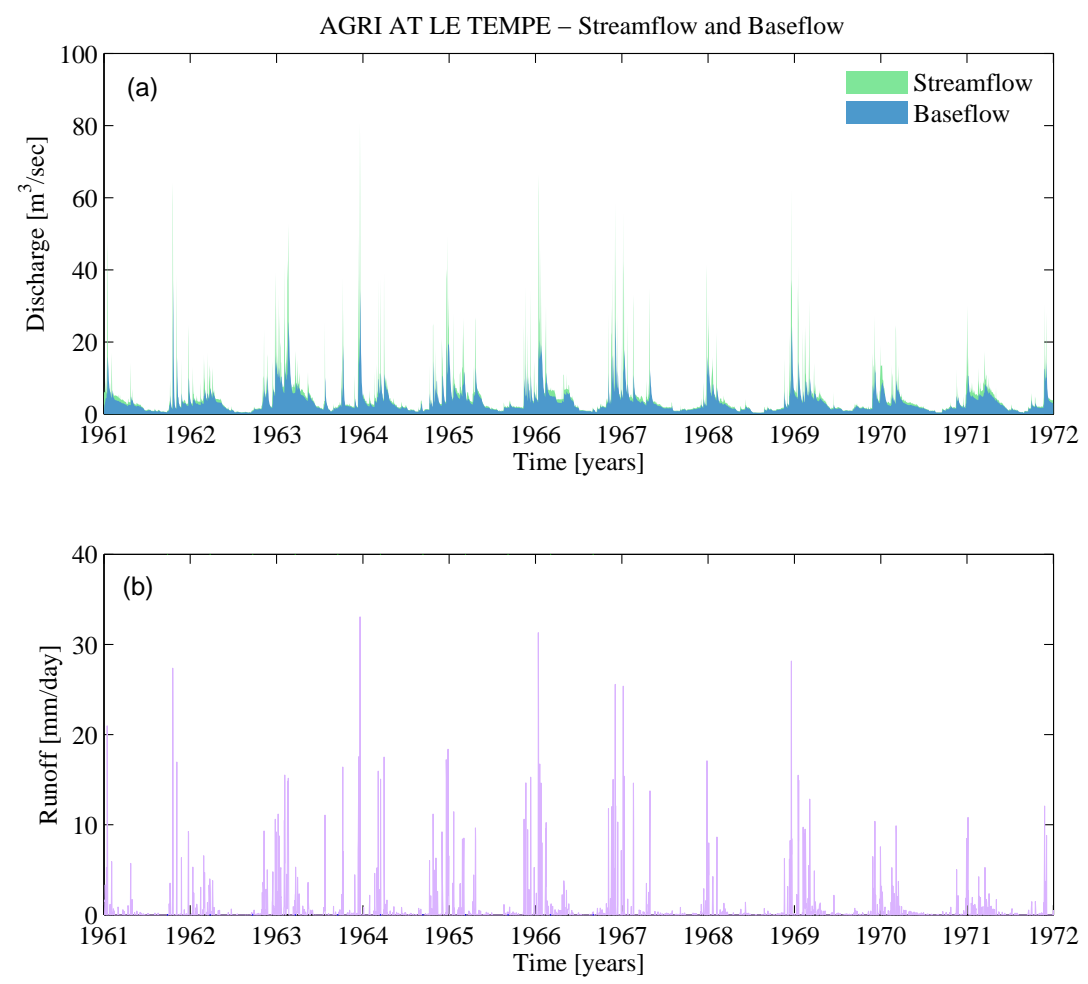

Fig. 4. Time series of the recorded streamflow over the period 1961-1971 at the station of "Le Tempe" of the Agri basin, a humid catchment of $174 \mathrm{~km}^{2}$ located in Southern Italy: (a) the graph describes the two components of the streamflow: the baseflow (colored in light blue) computed using the physically based filter proposed by Furey and Gupta (2001) and the second relative to the surface runoff (colored in green); (b) runoff production over the considered temporal window obtained as the difference between the recorded streamflow and the computed baseflow.

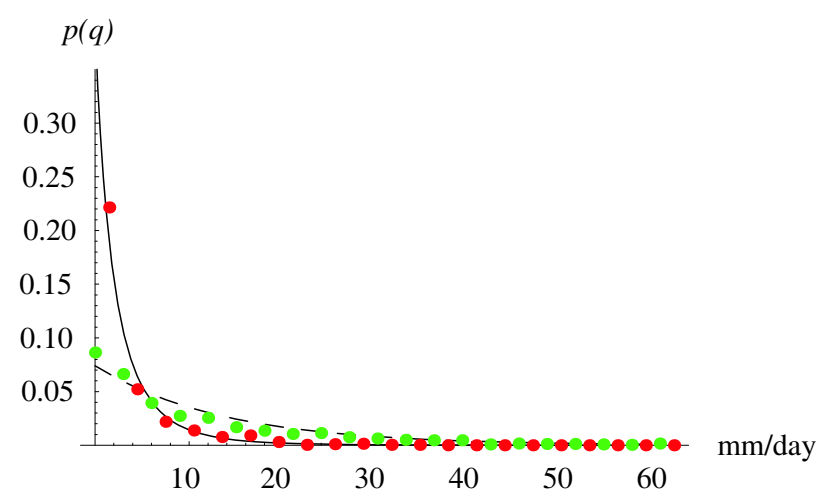

Fig. 5. Probability density functions of the runoff (red full circles) and the rainfall (green full circles) measured over the Agri basin at "Le Tempe" (Southern Italy) during the period 1961-1971. The continuous and the dashed lines represents the theoretical distribution of rainfall depths and runoff computed with the following parameters: $\lambda=0.43$ event/day, $\alpha=10.1 \mathrm{~mm}, V=6.24 \mathrm{~mm} /$ day, $b=0.39$ and finally $w_{\max }$ is assumed equal to $198 \mathrm{~mm}$.

\section{Results and discussion}

The probability density functions (PDFs) of runoff, obtained by derivation in $q$ of Eq. (19), are plotted in Fig. 3 using different set of parameters. In particular, PDFs are obtained adopting a given set of climatic and physical parameters changing the characteristics of the soil water storage capacity through the parameters maximum water storage capacity, $w_{\max }$, and exponent $b$ in the first and second graph of Fig. 3 (top-left and top-right). The third and fourth graphs describe the effects due to the soil water loss coefficient, $V$, and the rainfall rate, $\lambda$, on the runoff dynamics. In each of those graphs, the PDF of rainfall amount is also plotted in order to better understand the rainfall/runoff dynamics within the described scheme.

It can be noticed that the runoff production during a rainfall event is slightly affected by the maximum water storage capacity of the basin, but it is particularly sensitive to the spatial heterogeneity of the water storage capacity of the basin represented by the parameter $b$. This result is certainly due to the fact that the dynamics of expansion and contraction of the portion of saturated areas (source of runoff) is strongly controlled by the parameter $b$ (see Manfreda and Fiorentino, 2008). 
Among others, the parameter that seems to play a major role in the dynamics of runoff production is the coefficient of soil water loss that depends on evapotranspiration and leakage losses of the basin (Fig. 3 bottom-left). Finally, the parameter $\lambda$ also affects the runoff production being a controlling factor for the characteristic climate of the basin (Fig. 3 bottom-right).

\subsection{Model application to a real case}

The proposed probability distribution of runoff production may be used and applied to a real case to predict the dynamics of a hydrological system given the climatic forcing and the physical characteristics of the basin. In particular, parameters $\alpha$ and $\lambda$ can be easily defined from rainfall data at the daily scale, while the remaining parameters requires information on the soil permeability, vegetation cover, and topography.

In the present section, an application to a study basin of Southern Italy is presented in order to describe a possible estimation strategy for the model parameters. The challenge is to define those parameters starting from the available physical information of the basin avoiding to fit the theoretical distribution on the observed probability distribution of surface runoff.

The basin studied is the Agri basin closed at Le Tempe where a time series of 11 years of streamflow is available (see Fig. 4a). This record is necessary to test the model consistency in the description of runoff pruduction. It necessary to remark that the probability density functions refer to the surface runoff produced by the river basin and for this reason it is necessary to separate the baseflow component from the time series of streamflow. One possible strategy is to apply a mathematical filter to remove the baseflow component from the time series. The surface runoff data was estimated applying the physically-based mathematical filter proposed by Furey and Gupta (2001) to streamflow data considering the the overland flow coefficient, $c_{1}$, and the recharge coefficient, $c_{3}$, of the model variable from month to month following to the work of Manfreda et al. (2003). The results of the mathematical filter are displayed in Fig. 4 where the computed baseflow is displayed in light blue. The runoff time series are obtained as the difference between the streamflow and the baseflow during the days where a rainfall event occurred (see Fig. 4b).

Parameters of the theoretical distribution have been computed exploiting the available information on the Agri River basin. Rainfall parameters have been estimated from rainfall records during the wet season (October-March), the parameter $V$ is estimated from the equation obtained by Pan et al. (2003) for the Washita'92 dataset (Eq. 7) using the mean permeability $\left(K_{s}=2.40 \mathrm{~cm} / \mathrm{h}\right)$ of the basin and the mean Leaf Area Index $(\mathrm{LAI}=1.56)$ computed over the wet season.

The parameter $b$ was fitted using the method proposed by Chen et al. (2007) exploiting the TOPMODEL topo- graphic index, $W_{I}$, computed from a digital elevation model (DEM) at $240 \mathrm{~m}$ of resolution. The procedure is based on the hypothesis that the cumulative frequency distribution of the so called index of runoff generation difficulty $\left(\operatorname{IRDG}=\left(\max \left[W_{I}\right]-W_{I}\right) /\left(\max \left[W_{I}\right]-\min \left[W_{I}\right]\right)\right)$ can be used in place of the parabolic curve of soil water storage capacity of the Xinanjiang model. Under this hypothesis one can estimate the shape parameter $b$ fitting Eq. 2 with the cumulative frequency distribution of IRDG obtaining an estimate of $b=0.39$. Finally considering the small influenced played by the parameter $w_{\max }$, this last parameter was defined taking an average value in the range of variability (166 mm-222 mm) defined by Zhao (1984) and Zhao and Wang (1988).

The obtained probability distribution of surface runoff provides a realistic interpretation of the dynamics of a real river basin as one can appreciate comparing the theoretical function of rainfall and runoff to the ones obtained from data measured over the Agri River Basin at the station of Le Tempe (Fig. 5). This example is given not with the aim to fit the obtained probability distribution to the measured one, but only to demonstrate the consistency of the model outcomes when compared with a real case.

\section{Conclusions}

In the present paper, a new approach is introduced to describe analytically the relative soil saturation of a river basin and the runoff generation dynamics. The method provides a simplified description of river basin characteristics, but includes the effect of spatial variability of water storage capacity adopting the same schematization used by Zhao et al. (1980) for the Xinanjiang model.

The probability distribution of runoff describes in a feasible way the real dynamics of the system highlighting the role played by the different parameters controlling the soil water losses of a basin, the rate of rainfall and the characteristics of the soil water storage within a river basin. It was observed that the runoff production may be strongly controlled by the climate of the basin, while the spatial distribution of the soil water storage capacity plays a secondary role that apparently is more affected by the heterogeneity of soils that depends on the exponent $b$ of Eq. (2) rather than the maximum water storage capacity, $w_{\max }$.

In the present model the soil water storage distribution is assumed as a parabolic function with a minimum in zero. This imply that the relative saturated areas that are responsible of the runoff production are always higher than zero. Under this assumption the rate of runoff is equal to the rate of rainfall that is a realistic assumption for humid environments. Nevertheless, the approach can be generalized adopting a parabolic function translated of a constant value in order to obtain a distribution of soil depths bounded between two non zero-values. 
6 Notation

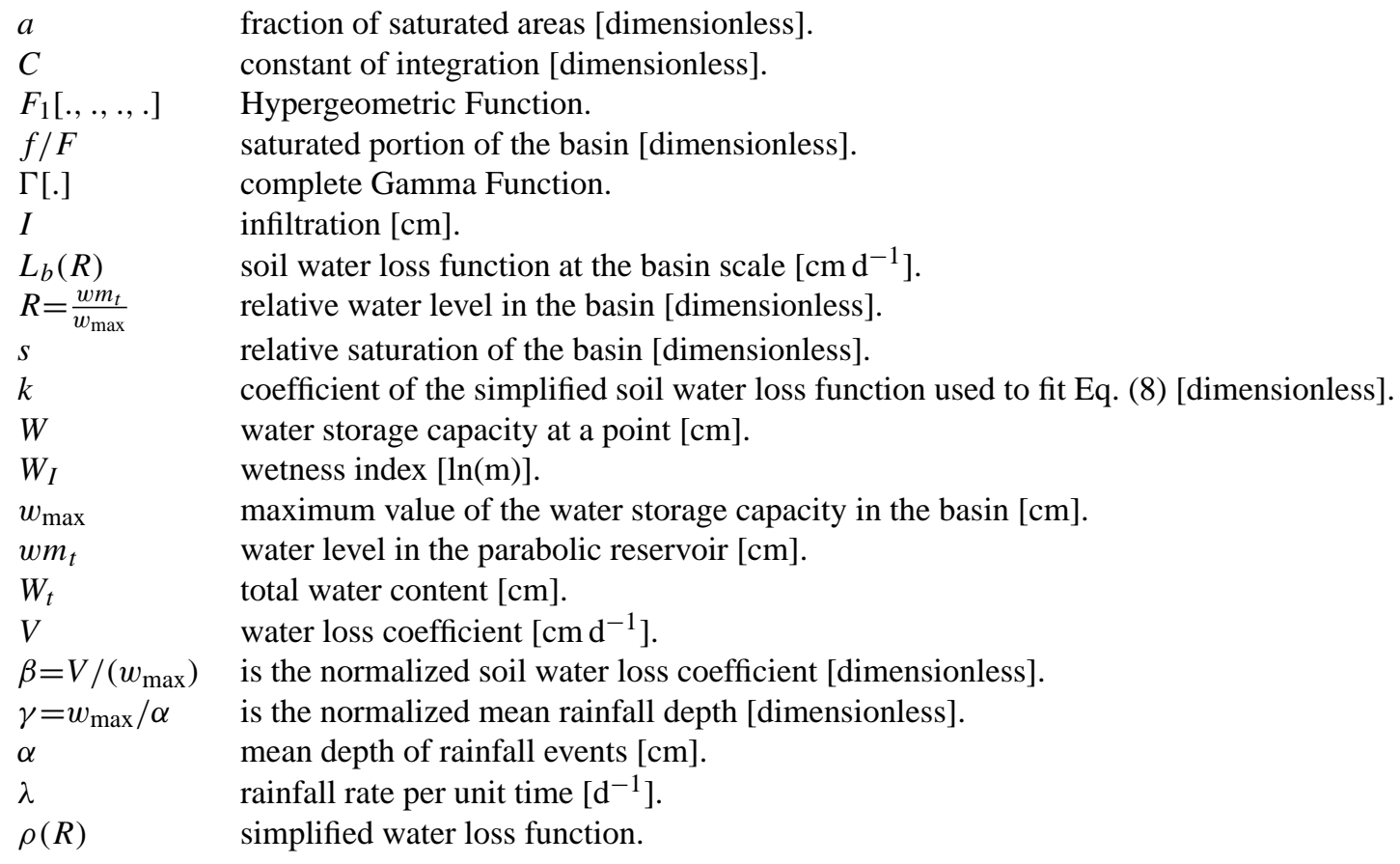

Acknowledgements. Salvatore Manfreda gratefully acknowledge the support of MIUR (Italian Ministry of Instruction, University and Research) under the grant PRIN CoFin2007 entitled "Spatialtemporal patterns in hydrological and ecological processes" and of the CARICAL foundation for his research activities.

Edited by: G. Roth

Reviewed by: two anonymous referees

\section{References}

Abramowitz, M. and Stegun, I. A.: Handbook of Mathematical Functions with Formulas, Graphs, and Mathematical Tables, New York, Dover, 1046 pp., 1964.

Arnold, L.: Stochastic Differential Equations: Theory and Applications, John Wiley \& Sons, New York, 228 pp., 1974.

Benjamin, J. R. and Cornell, C. A.: Probability, Statistics and Decisions for Civil Engineers, McGraw Hill, New York, 640 pp., 1970.

Chen, X., Chen, Y. D., and Xu, C.-Y.: A distributed monthly hydrological model for integrating spatial variations of basin topography and rainfall, Hydrol. Process., 21(2), 242-252, doi:10.1002/hyp.6187, 2007.

Dunne, T. and Black, R.: An Experimental Investigation of Runoff Production in Permeable Soils, Water Resour. Res., 6(2), 478490, 1970

Entekhabi, D. and Rodríguez-Iturbe, I.: An analytic framework for the characterization of the space-time variability of soil moisture, Adv. Water Resour., 17, 25-45, 1994.

Furey, P. R. and Gupta, V. K.: A physically based filter for separating base flow from streamflow time series, Water Resour. Res., 37(11), 2709-2722, 2001.
Hewlett, J. D. and Hibbert, A. R.: Factors affecting the response of small watersheds to precipitation in humid areas, in: Forest Hydrology, edited by: Sopper, W. E. and Lull, H. W., Pergamon Press, 275-290, 1967.

Horton, R. E.: The role of infiltration in the hydrologic cycle, Trans. Am. Geophys. Union, 14, 446-460, 1933.

Isham, V., Cox, D. R., Rodríguez-Iturbe, I., Porporato, A., and Manfreda, S.: Mathematical characterization of the space-time variability of soil moisture, P. Roy. Soc. A.-Math. Phy., 461(2064), 4035-4055, 2005.

Laio, F., Porporato, A., Fernandez-Illescas, C. P., and RodríguezIturbe, I.: Plants in water-controlled ecosystems: Active role in hydrologic processes and response to water stress, IV: Discussion of real cases, Adv. Water Resour., 24, 745-762, 2001.

Liang, X., Lettenmaier, D. P., Wood, E. F., and Burges, S. J.: A simple hydrologically based model of land surface water and energy fluxes for GCMs, J. Geophys. Res., 99(D7), 14 415-14 428, 1994.

Manfreda, S. and Fiorentino, M.: A stochastic approach for the description of the water balance dynamics in a river basin, Hydrol. Earth Syst. Sci., 12, 1189-1200, 2008, http://www.hydrol-earth-syst-sci.net/12/1189/2008/.

Manfreda, S., Giordano, C., Iacobellis, V.: Stima dei Deflussi di Base mediante un Filtro Fisicamente Basato, Proocidings of "Giornata di Studio: Metodi Statistici e Matematici per l'Analisi delle Serie Idrologiche" edited by: Piccolo, D. and Ubertini, L., ISBN: 88-88885-00-5, 247-258, 2003 (in Italian).

Manfreda, S. and Rodríguez-Iturbe, I.: On the Spatial and Temporal Sampling of Soil Moisture Fields, Water Resour. Res., 42, W05409, doi:10.1029/2005WR004548, 2006.

Moore, R. J. and Clarke, R. T.: A distribution function approach to rainfall runoff modelling, Water Resour. Res., 17, 1367-1382, 1981.

Moore, R. J.: The probability-distributed principle and runoff pro- 
duction at point and basin scales, Hydrol. Sci., 30, 273-297, 1985.

Moore, R. J.: Real-time flood forecasting system: perspectives and prospects, in: Flood and landslides: Integrated risk assessment, edited by: Casal, R. and Margottini C., Springer, 147-189, 1999.

Pan, F., Peters-Lidard, C. D., and Sale, M. J.: An analytical method for predicting surface soil moisture from rainfall observations, Water Resour. Res., 39(11), 1314, doi:10.1029/2003WR002142, 2003.

Porporato, A., Daly, E., and Rodríguez-Iturbe, I.: Soil water balance and ecosystem response to climate change, Am. Nat., 164(5), 625-633, 2004.

Rodríguez-Iturbe, I., Porporato, A., Ridolfi, L., Isham, V., and Cox, D. R.: Probabilistic modelling of water balance at a point: The role of climate, soil and vegetation, P. Roy. Soc. A.-Math. Phy., 455, 3789-3805, 1999.

Todini, E.: The ARNO rainfall-runoff model, J. Hydrol., 175, 339-
382, 1996.

Wood, E. F., Lettenmaier, D. P., Liang, X., Nijssen, B., and Wetzel, S. W.: Hydrological modeling of continental-scale basins, Annu. Rev. Earth Pl. Sc., 25, 279-300, 1997.

Wood, E. F., Lettenmaier, D. P., and Zartarian, V. G.: A land-surface hydrology parametrization with subgrid varibility for general circulation models, J. Geophys. Res., 97, 2717-2728, 1992.

Zhao, R. J. and Wang, P. L.: Parameter determination of Xinanjiang model, Hydrology, 6, 2-9, 1988 (in Chinese).

Zhao, R.-J., Zhang, Y. L., and Fang, L. R.: The Xinanjiang model, Hydrological Forecasting Proceedings Oxford Symposium, IAHS Pub. 129, 351-356, 1980.

Zhao, R.-J.: The Xinanjiang model applied in China, J. Hydrol., 135, 371-381, 1992.

Zhao, R.-J.: Water Hydrological Modeling-Xinanjiang Model and Shanbei Model, China Water Resources and Hydropower Publishing House, Beijing, 125-129, 1984 (in Chinese). 